Journal of Organometallic Chemistry, 347 (1988) 237-252

Elsevier Sequoia S.A., Lausanne - Printed in The Netherlands

\title{
Reaktivität der Metall-Metall-Mehrfachbindung in Übergangsmetall-Komplexen
}

\section{XIII *. Untersuchungen zum stufenweisen Aufbau chalkogenreicher Zweikernkomplexe durch Reaktion von $\left[\mathrm{Cp}^{\star} \mathrm{M}(\mathrm{CO})\right]_{2}\left(\mathrm{M}=\mathrm{Co}, \mathrm{Rh} ; \mathrm{Cp}^{\star}=\eta^{5}-\mathrm{C}_{5} \mathrm{Me}_{5}\right)$ mit elementarem Schwefel, Selen oder Tellur}

\author{
Henri Brunner, Norbert Janietz, Walter Meier, Joachim Wachter *, \\ Institut für Anorganische Chemie der Universität Regensburg, Universitätsstrasse 31, \\ D-8400 Regensburg (B.R.D.)
}

Eberhard Herdtweck, Wolfgang A. Herrmann,

Anorganisch-Chemisches Institut der Technischen Universität München, Lichtenbergstrasse 4, D-8046 Garching (B.R. D.)

Omar Serhadli und Manfred L. Ziegler

Anorganisch-Chemisches Institut der Universität Heidelberg, Im Neuenheimer Feld 270,

D-6900 Heidelherg (B.R.D.)

(Eingegangen den 10. Dezember 1987)

\begin{abstract}
Reaction of the complexes $\mathrm{Cp}^{\star}{ }_{2} \mathrm{M}_{2}(\mu-\mathrm{CO})_{2}\left(M=M ; \mathrm{Cp}^{\star}=\eta^{5}-\mathrm{C}_{5} \mathrm{Me}_{5} ; \mathrm{M}=\mathrm{Co}\right.$, Rh) with elemental sulfur, sclenium, or tellurium results in addition of one or two equivalents of the chalcogen to the $\mathbf{M}-\mathbf{M}$ double bond, the outcome is dependent on the reaction conditions and the stoichiometry. Complexes of the type $\mathrm{Cp}^{\star}{ }_{2} \mathrm{Rh}_{2}(\mathrm{CO})_{2} \mathrm{E}(\mathrm{E}=\mathrm{S}, \mathrm{Se})$ and $\mathrm{Cp}^{\star}{ }_{2} \mathrm{M}_{2}(\mathrm{CO})_{2} \mathrm{E}_{2}(\mathrm{M}=\mathrm{Co}, \mathrm{Rh} ; \mathrm{E}=\mathrm{Se}, \mathrm{Te})$ have been isolated and characterized spectroscopically. Reaction of excess chalcogen with $\mathrm{Cp}^{\star}{ }_{2} \mathrm{M}_{2}(\mathrm{CO})_{2}$ gives the CO-free diamagnetic compounds $\mathrm{Cp}_{2}{ }_{2} \mathrm{Co}_{2} \mathrm{~S}_{4}, \mathrm{Cp}^{\star}{ }_{2} \mathrm{Rh}_{2} \mathrm{~S}_{8}$, and $\mathrm{Cp}^{\star}{ }_{2} \mathrm{M}_{2} \mathrm{Se}_{5}(\mathrm{M}=\mathrm{Co}, \mathrm{Rh})$. X-ray diffraction studies have been carried out on $\mathrm{Cp}^{\star}{ }_{2} \mathrm{Co}_{2} \mathrm{Se}_{5}$ and $\mathrm{Cp}_{2}^{\star}{ }_{2} \mathrm{Rh}_{2}(\mathrm{CO})_{2} \mathrm{Se}$. The latter compound exhibits a $\mathrm{Rh}_{2} \mathrm{Se}$ threemembered cycle with attached anti-oriented $\mathrm{CP}^{\star}$ and $\mathrm{CO}$ ligands. In the cobalt compound a $\mathrm{Se}^{2-}$ and a $\mathrm{Se}_{4}{ }^{2-}$ ligand form a nearly planar pseudo five-membered
\end{abstract}

* XII. Mitteilung siehe Ref. 1. 
cycle, which bisects the Co-Co axis. All reactions can be interpreted in terms of stepwise addition of chalcogens to the $\mathrm{M}-\mathrm{M}$ double bond with subsequent replacement of carbon monoxide.

\section{Zusammenfassung}

Die Reaktion der Komplexe $\mathrm{Cp}^{\star}{ }_{2} \mathrm{M}_{2}(\mu-\mathrm{CO})_{2}\left(M=M ; \mathrm{Cp}^{\star}=\eta^{5}-\mathrm{C}_{5} \mathrm{Me}_{5} ; \mathrm{M}=\mathrm{Co}\right.$, Rh) mit elementarem Schwefel, Selen oder Tellur führt je nach Reaktionsbedingungen und Stöchiometrie zur Addition von ein oder zwei Äquivalenten Chalkogen an die M-M-Doppelbindung. Komplexe des Typs $\mathrm{Cp}^{\star}{ }_{2} \mathrm{Rh}_{2}(\mathrm{CO})_{2} \mathrm{E}(\mathrm{E}=\mathrm{S}, \mathrm{Se})$ und $\mathrm{Cp}_{2}{ }_{2} \mathrm{M}_{2}(\mathrm{CO})_{2} \mathrm{E}_{2}(\mathrm{M}=\mathrm{Co}, \mathrm{Rh} ; \mathrm{E}=\mathrm{Se}, \mathrm{Te})$ konnten dabei in Substanz isoliert und spektroskopisch charakterisiert werden. Die Reaktion von überschüssigem Chalkogen mit $\mathrm{CP}^{\star}{ }_{2} \mathrm{M}_{2}(\mathrm{CO})_{2}$ führt zu den $\mathrm{CO}$-freien diamagnetischen Verbindungen $\mathrm{Cp}^{\star}{ }_{2} \mathrm{Co}_{2} \mathrm{~S}_{4}, \mathrm{Cp}^{\star}{ }^{\star} \mathrm{Rh}_{2} \mathrm{~S}_{8}$ und $\mathrm{Cp}^{\star}{ }_{2} \mathrm{M}_{2} \mathrm{Se}_{5}(\mathrm{M}=\mathrm{Co}, \mathrm{Rh})$. Röntgenstrukturanalysen wurden von $\mathrm{Cp}^{\star}{ }_{2} \mathrm{Co}_{2} \mathrm{Se}_{5}$ und $\mathrm{Cp}^{\star}{ }_{2} \mathrm{Rh}_{2}(\mathrm{CO})_{2} \mathrm{Se}$ angefertigt. Letztere Verbindung ist durch einen $\mathrm{Rh}_{2}$ Se-Dreiring und anti-ständige $\mathrm{Cp}^{\star}$ - und CO-Liganden charakterisiert. Im Co-Komplex bilden ein $\mathrm{Se}^{2-}$ - und ein $\mathrm{Se}_{4}{ }^{2-}$-Ligand einen nahezu planaren, die Co-Co-Achse halbierenden Pseudofünfring. Sämtliche Reaktionen lassen sich als stufenweise Addition der Chalkogene an die M-M-Doppelbindung mit nachfolgender CO-Substitution deuten.

\section{Einleitung}

Einen einfachen Zugang zu den schwefelreichen Zweikernkomplexen $\mathrm{Cp}^{\star}{ }_{2} \mathbf{M}_{2} \mathrm{~S}_{x}$ $(x=4,5 ; \mathrm{M}=\mathrm{Cr}, \mathrm{Mo}, \mathrm{W}$ ) bietet die Reaktion der $\mathbf{M}$-M-Dreifachbindung der Komplexe $\mathrm{Cp}^{\star}{ }_{2} \mathrm{M}_{2}(\mathrm{CO})_{4}\left(\mathrm{Cp}^{\star}=\eta^{5}-\mathrm{C}_{5} \mathrm{Me}_{5}\right)[2,3]$ mit Schwefel. Der vor allem durch Isomerie bedingten Vielfalt dieser Verbindungen - es liegen sowohl Monoals auch Dischwefelliganden, aber auch beide kombiniert vor - steht mit der Verbindung $\mathrm{Cp}^{\star}{ }_{2} \mathrm{Mo}_{2}\left(\mu-\mathrm{Se}_{2}\right)(\mu-\mathrm{Se})_{2}$ nur ein einziger rein selenhaltiger Komplex gegenüber [4].

Im Gegensatz hierzu zeigt die M-M-Doppelbindung der Komplexe $\mathrm{Cp}_{2}^{\star}{ }_{2} \mathrm{M}_{2}(\mathrm{CO})_{2}$ $(M=\mathrm{Co}, \mathrm{Rh})$ eine ausgeprägte Reaktivität auch gegenüber Selen und Tellur [1]. In dieser Arbeit zeigen wir, dass Stöchiometrie und Stabilität der gebildeten Produkte sowohl vom eingesetzten Chalkogen als auch von der Natur des Übergangsmetalls bestimmt werden. Dies erlaubt nicht nur einen tieferen Einblick in den Bildungsmechanismus chalkogenreicher Übergangsmetallkomplexe, sondern vermittelt auch Aufschlüsse über den Abbau der eingesetzten Chalkogenringe bzw. -ketten. Die hierbei u. a. gebildeten Komplexe $\mathrm{Cp}^{\star}{ }_{2} \mathrm{Rh}_{2} \mathrm{~S}_{8}$ bzw. $\mathrm{Cp}^{\star}{ }_{2} \mathrm{Rh}_{2} \mathrm{Se}_{5}$ scheinen die stabilen Endprodukte dieses Abbaus zu sein. Über sie wurde bereits in Kurzmitteilungen berichtet $[1,5]$.

\section{Die Reaktion von $\mathrm{Cp}_{2}^{\star} \mathrm{M}_{2}(\mathrm{CO})_{2}(\mathrm{M}=\mathrm{Co}, \mathrm{Rh})$ mit Schwefel}

Die Reaktion von $\mathrm{Cp}_{2}{ }_{2} \mathrm{Co}_{2}(\mathrm{CO})_{2}$ mit Cyclooctaschwefel in Toluol ergibt unabhängig von der Stöchiometrie als einziges isolierbares Produkt den grünen, carbonylfreien Komplex $\mathrm{Cp}^{\star}{ }_{2} \mathrm{Co}_{2} \mathrm{~S}_{4}$ (III), der durch zwei $\mu, \eta^{1}, \eta^{2}-\mathrm{S}_{2}$-Liganden charakterisiert ist [6]. Selbst bei Schwefelunterschuss konnten CO-haltige Produkte 
IR-spektroskopisch nur in Lösung beobachtet ( $\boldsymbol{\nu}(\mathrm{CO})-$ Frequenzen bei 2000 bzw. $1940 \mathrm{~cm}^{-1}$, Toluollösung) werden.

Im Gegensatz hierzu reagiert $\mathrm{Cp}_{2}^{\star} \mathrm{Rh}_{2}(\mathrm{CO})_{2}$ bereits mit einem Äquivalent Schwefel (THF, $20^{\circ} \mathrm{C}$ ) in guter Ausbeute zu Cp ${ }_{2}^{\star} \mathrm{Rh}_{2}(\mathrm{CO})_{2} \mathrm{~S}$ (IV). Die analytischen und spektroskopischen Daten (IR: $\nu(C O) 1955 \mathrm{~cm}^{-1} ;{ }^{1} \mathrm{H}-\mathrm{NMR}: \delta=1.93$ $\left(\mathrm{CDCl}_{3}\right)$ ) sind vereinbar mit der Addition eines Schwefelatoms an die $\mathbf{R h}=\mathbf{R h}-\mathrm{Bin}-$ dung unter gleichzeitiger Öffnung der Carbonylbrücken. Der Versuch, ein weiteres Äquivalent Schwefel an IV zu addieren, führt bei Raumtemperatur zur Bildung des CO-freien, überraschend schwefelreichen Komplexes $\mathrm{Cp}^{\star}{ }_{2} \mathrm{Rh}_{2} \mathrm{~S}_{8}$ (VII) neben unverbrauchtem IV. Verfolgt man diese Reaktion IR-spektroskopisch zwischen -60 und $-80^{\circ} \mathrm{C}$, so beobachtet man zwar die Abnahme der für IV typischen $\mathrm{CO}-\mathrm{Ab}-$ sorption bei $1955 \mathrm{~cm}^{-1}$, jedoch wächst gleichzeitig eine neue Bande bei $1811 \mathrm{~cm}^{-1}$. Diese ist für den CO-verbrückten Komplex VI typisch. Weitere CO-Absorptionen im Bereich zwischen 1800 und $2000 \mathrm{~cm}^{-1}$ sind sehr schwach und verschwinden mit

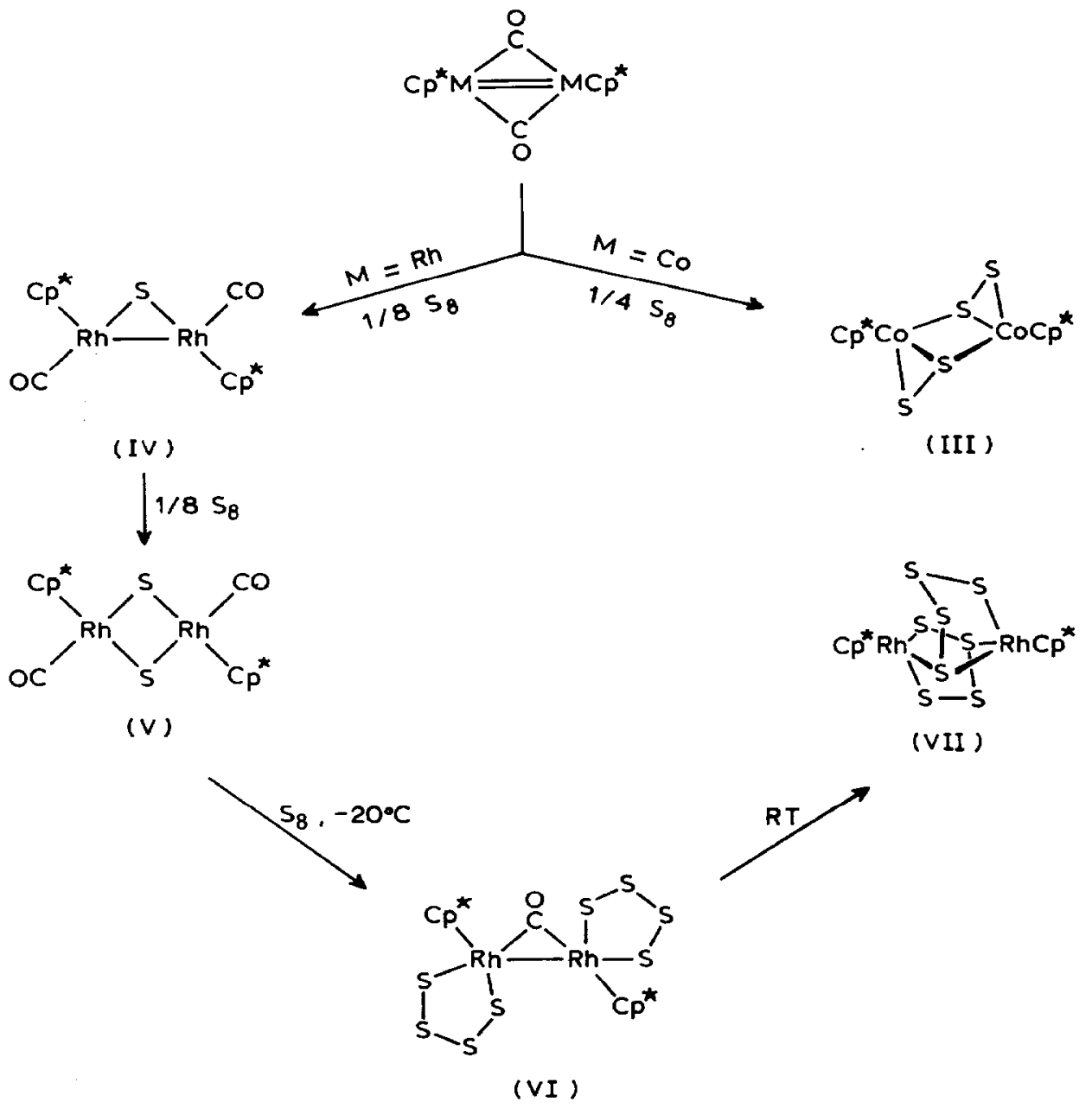

Schema 1. Die Reaktion von $\mathrm{Cp}^{\star}{ }_{2} \mathrm{M}_{2}(\mathrm{CO})_{2}\left(\mathrm{Cp}^{\star}=\eta^{5}-\mathrm{C}_{5} \mathrm{Me}_{5}, \mathrm{M}=\mathrm{Co}\right.$ : I; $\mathrm{M}=\mathrm{Rh}$ : II $)$ mit Schwefel. 
zunehmender Reaktionsdauer wieder. Für das eigentlich erwartete Diadditionsprodukt $\mathrm{V}$ gibt es nur den Hinweis einer schwachen Bande bei $2015 \mathrm{~cm}^{-1}$.

Eine optimale Ausbeute an VI wird bei $-20^{\circ} \mathrm{C}$ und Steigerung des S/RhVerhältnisses auf $4 / 1$ erreicht (Schema 1). Unter diesen Bedingungen entsteht jedoch neben VI auch noch $\mathrm{Cp}^{\star}{ }_{2} \mathrm{Rh}_{2} \mathrm{~S}_{8}$ (VII) in ungefähr gleichem Verhältnis.

Die Bildung von VI wurde bereits früher beobachtet [7]. Analytische Daten sowie IR-Spektrum ( $\nu(\mathrm{CO}) 1811 \mathrm{~cm}^{-1}$ ) führten zur Annahme von zwei $\mathrm{RhS}_{4}$-Chelaten, die über eine CO-Brücke sowie direkt über eine $\mathbf{R h}-\mathrm{Rh}$-Bindung miteinander verknüpft sein sollten [8]. Der symmetrische Aufbau wird nunmehr auch durch die Präsenz von nur einer Methylresonanz im ${ }^{1} \mathrm{H}-\mathrm{NMR}$-Spektrum bestätigt. VI ist in Lösung labil, was bei Raumtemperatur unter langsamem CO-Verlust zu VII führt. VII ist auch direkt durch Reaktion von II mit leichtem Schwefelüberschuss und verlängerter Reaktionsdauer (15-17 h) in schr guten Ausbeuten (bis zu 69\%) erhältlich. Die Molekülstruktur von VII [5] enthält zwei $\mathbf{R h S}_{4}$-Chelate, die so untereinander verknüpft sind, dass sie einen leicht gewellten $R h_{2} S_{3}$-Fünfring bilden. Die freien, nicht koordinierten Schwefelglieder sind anti-ständig orientiert. Dieser Befund bestätigt somit im Nachhinein die Annahme zweier nichtverbrückender $\mathrm{RhS}_{4}$-Chelate in VI. Wahrscheinlich sind sterische Gründe dafür verantwortlich, dass einmal das $\alpha$-ständige Schwefelatom des einen Rings bzw. das $\beta$-ständige Schwefelatom des anderen Rings am jeweils gegenüberliegenden $R h-Z e n t r u m$ angreifen kann, wodurch der $\mathrm{Rh}_{2} \mathrm{~S}_{3}$-Kern von VII entsteht.

\section{Die Reaktion von $\mathrm{Cp}_{2}^{\star}{ }_{2} \mathrm{M}_{2}(\mathrm{CO})_{2}(\mathrm{M}=\mathrm{Co}$, Rh) mit Selen und Tellur}

Monoaddukte der allgemeinen Zusammensetzung $\mathrm{Cp}^{\star}{ }_{2} \mathrm{M}_{2}(\mathrm{CO})_{2} \mathrm{E}$ lassen sich nur für $M=R h$ und $E=$ Se darstellen. Diese Reaktion wurde bereits einmal verfolgt mit dem Ziel, eine hochreaktive $\mathrm{Rh}=\mathrm{Se}=\mathrm{Rh}$-Struktureinheit aufzubauen: Die $\mathrm{THF}$ Lösung von II reagiert bei Raumtemperatur in Minutenfrist mit grauem Selen zu $\mathrm{Cp}^{\star}{ }_{2} \mathrm{Rh}_{2}$ (CO) ${ }_{2} \mathrm{Se}$ (VIII) [9].

Die Diaddukte $\mathrm{Cp}^{\star}{ }_{2} \mathrm{M}_{2}(\mathrm{CO})_{2} \mathrm{Se}_{2}$ (IXa,b) lassen sich durch Reaktion von I (Toluol, $0^{\circ} \mathrm{C}$ ) bzw. II oder VIII $\left(50^{\circ} \mathrm{C}\right)$ mit zwei Äquivalenten grauem Selen darstellen (Schema 2). In beiden Fällen ist nach beendeter Reaktion die sofortige Entfernung unverbrauchten Selens durch Filtration der Reaktionslösung über $\mathrm{Al}_{2} \mathrm{O}_{3}$

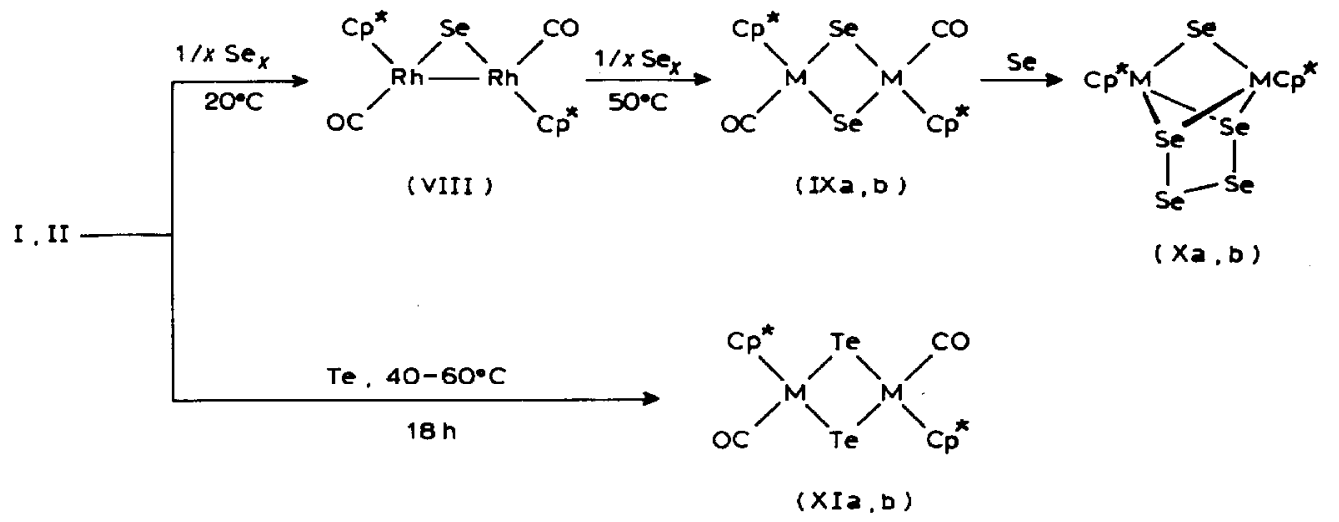

Schema 2. Reaktion von $\left[C \mathrm{p}^{\star}(\mathrm{CO}) \mathrm{M}\right]_{2}$ mit Selen und Tellur $(\mathrm{M}=\mathrm{Co}$ : $\mathrm{a}, \mathbf{M}=\mathrm{Rh}$ : b). 
erforderlich, da sonst CO-freie Produkte gebildet werden. Die Darstellung der Tellurkomplexe XIa,b erfordert dagegen neben erhöhten Temperaturen $\left(40-60^{\circ} \mathrm{C}\right)$ noch drastisch verlängerte Reaktionszeiten (18 h).

Die CO-freien Komplexe Xa,b lassen sich sowohl aus VIII als auch aus IXa,b durch Reaktion mit Selenüberschuss gewinnen. Optimale Ausbeuten erzielt man jedoch durch direkten Umsatz von I (80\%) bzw. II (19\%) bei entsprechend verlängerter Reaktionszeit (Toluol, $18 \mathrm{~h}$, Raumtemperatur). Totalsubstitution der CO-Liganden lässt sich zwar auch mit Tellur erreichen $\left(100^{\circ} \mathrm{C}, 68 \mathrm{~h}\right)$, die laut ${ }^{1} \mathrm{H}$-NMR resultierenden Produktgemische lassen sich jedoch nicht in ihre Einzelbestandteile auftrennen.

\section{Der Strukturtyp $\mathrm{Cp}_{2}^{\star} \mathrm{M}_{2}(\mathrm{CO})_{2} \mathrm{E}$}

Chalkogenbrïckenliganden $\mathrm{E}$ sind in besonderem Masse zur Stabilisierung von Metall-d-Bahnfunktionen über $\pi$-Wechselwirkungen befähigt [10]. Dies sollte im vorliegenden Fall zu linearen oder gewinkelten $\mathrm{M}-\mathrm{E}-\mathrm{M}-\mathrm{Mehrfachbindungssyste-}$ men ohne $\mathrm{M}-\mathrm{M}$-Bindung führen. Auch $\mathrm{Cp}_{2}^{\star} \mathrm{Rh}_{2}(\mathrm{CO})_{2}$ Se wurde zunächst in dem Bestreben synthetisiert, ein kumuliertes $\mathbf{R h}-\mathrm{Se}-\mathrm{Rh}-\mathrm{Mehrfachbindungssystem} \mathrm{von}$ hoher Reaktivität aufzubauen [9]. ${ }^{13} \mathrm{C}-\mathrm{NMR}$-spektroskopische Untersuchungen belegen einen intramolekularen Austausch der CO-Gruppen und sprechen somit für die Existenz einer $\mathbf{R h}-\mathbf{R h}-B i n d u n g$. Dagegen enthält das ${ }^{77}$ Se-NMR-Spektrum einen Hinweis auf die Präsenz von $\mathbf{R h}$-Se-Mehrfachbindungsanteilen: In $\mathrm{CHCl}_{3}$-Lösung $\left(0^{\circ} \mathrm{C}\right)$ wird ein relativ breites $(200 \mathrm{~Hz})$ Resonanzsignal bei $891.8 \mathrm{ppm}$ gefunden, was eine Tieffeldverschiebung von $406.6 \mathrm{bzw} .108 .7 \mathrm{ppm}$ gegenüber den in $\mathrm{Cp}^{\star}{ }_{2} \mathrm{Rh}_{2} \mathrm{Se}_{5}$ gefundenen Resonanzen [1] bedeutet. Ähnlich tiefe Se-Resonanzsignale wurden bisher nur für den 16-e-Komplex $\left(\mathrm{t}-\mathrm{BuC}_{5} \mathrm{H}_{4}\right)_{4} \mathrm{Zr}_{2}(\mu-\mathrm{Se})_{2}$ beobachtet $\left[11,12^{*}\right]$.

Zur endgültigen Klärung dieser Frage wurde an Einkristallen von VIII eine Röntgenstrukturanalyse durchgeführt. Die Atomkoordinaten und wichtige Bindungsparameter sind in den Tab. 1 und 2 aufgeführt. Als prinzipielles Strukturelement enthält VIII einen $R_{h_{2}}$ Se-Dreiring (Fig. 1). Die CO- und $C_{5} M_{5}$-Liganden sind hierzu anti-ständig angeordnet. Auf Grund dieser Anordnung lässt sich VIII nunmehr zwanglos in die isoelektronische Serie von Dimetallacyclopropanen ( $\mu$ $\mathrm{X})[\mathrm{CpRh}(\mathrm{CO})]_{2}\left(\mathrm{X}=\mathrm{CH}_{2}\right.$ [13], $\mathrm{CO}$ [13], $\mathrm{SO}_{2}$ [14]) einreihen. Bedingt durch die Grösse des Selenatoms wird jedoch die Dreiringgeometrie merklich beeinflusst, was sich in einer geringfügigen Aufweitung der $\mathbf{R h}-\mathrm{Rh}$-Bindung und dem bisher kleinsten gefundenen $\mathrm{Rh}-\mathrm{X}-\mathrm{Rh}-$ Winkel $\left(68.7(<1)^{\circ}\right)$ ausdrückt. Dagegen ist das $\mathbf{R h}-\mathrm{Se}-\mathrm{Rh}$-Strukturelement in $\mathrm{Cp}^{\star}{ }_{2} \mathbf{R h}_{2} \mathrm{Se}_{5}$ - einem Komplex, der ausschliesslich durch Selenbrücken verknüpft ist - durch einen Winkel von $86.6(1)^{\circ}$ charakterisiert [1]. Der $R h-S e-A b s t a n d\left(2.440(<1) \AA\right.$ ) ist nur geringfügig kürzer als in $C^{\star}{ }_{2}{ }_{2} R_{2} S_{2} e_{5}$ (2.460-2.481 $\AA$ ) [1] oder in [(triphos) $\left.{ }_{2} \mathrm{Rh}_{2}\left(\mu, \eta^{1}, \eta^{2}-\mathrm{Se}_{2}\right)_{2}\right]\left[\mathrm{PPh}_{4}\right]_{2}(2.494,2.531(1) \AA)$ [15].

Ebenso wie VIII sollte auch dessen S-Homolog IV zum Strukturtyp A gehören. Dieser wird generell für $\mathbf{M}_{2}$ E-Komplexe mit $\mathrm{CO}$ - und $\mathrm{Cp}-\mathrm{Liganden}$ der 2 . und 3. Periode beobachtet. Im Gegensatz hierzu scheinen die $3 d$-Metalle zur Bildung "offener" Strukturen vom Typ B zu neigen, was durch das unterschiedliche Verhalten der Komplexe $\left[\mathrm{Cp}(\mathrm{CO})_{2} \mathrm{Mn}\right]_{2} \mathrm{~S}\left[16^{*}\right]$ (Typ B) und $\left[\mathrm{Cp}(\mathrm{CO})_{2} \operatorname{Re}\right] \mathrm{S}$ [17] (Typ A)

\footnotetext{
* Die Literaturnummer mit einem Sternchen deutet eine Bemerkung in der Literaturliste an.
} 
Tabelle 1

Atomkoordinaten von $\mathrm{Cp}_{2}^{\star} \mathrm{Rh}_{2}(\mathrm{CO})_{2} \mathrm{Se}$ (VIII)

\begin{tabular}{|c|c|c|c|c|}
\hline Atom & $x / a$ & $y / b$ & $z / c$ & $B\left(\AA^{2}\right)^{a}$ \\
\hline$\overline{\mathbf{R h}}$ & $0.52251(2)$ & $0.66527(1)$ & $0.15904(2)$ & $2.754(4)$ \\
\hline $\mathrm{Se}$ & 0.500 & $0.52128(3)$ & 0.250 & $3.459(9)$ \\
\hline $\mathbf{O}$ & $0.2674(2)$ & $0.6581(2)$ & $0.0813(2)$ & $6.03(6)$ \\
\hline $\mathrm{C}$ & $0.3639(3)$ & $0.6615(2)$ & $0.1161(2)$ & $3.85(7)$ \\
\hline $\mathrm{C}(11)$ & $0.7198(2)$ & $0.6728(2)$ & $0.1559(2)$ & $3.43(6)$ \\
\hline$C(12)$ & $0.6606(3)$ & $0.6396(2)$ & $0.0674(2)$ & $3.61(7)$ \\
\hline$C(13)$ & $0.5828(3)$ & $0.7128(3)$ & $0.0288(2)$ & $4.20(7)$ \\
\hline $\mathrm{C}(14)$ & $0.5936(3)$ & $0.7918(2)$ & $0.0923(2)$ & $4.22(7)$ \\
\hline$C(15)$ & $0.6807(3)$ & $0.7680(2)$ & $0.1703(2)$ & $3.66(7)$ \\
\hline$C(21)$ & $0.8160(3)$ & $0.6192(3)$ & $0.2165(3)$ & $4.84(8)$ \\
\hline $\mathrm{C}(22)$ & $0.6827(3)$ & $0.5459(3)$ & $0.0230(3)$ & $5.93(9)$ \\
\hline$C(23)$ & $0.5094(3)$ & $0.7090(4)$ & $-0.0662(3)$ & $6.8(1)$ \\
\hline$C(24)$ & $0.5325(3)$ & $0.8871(3)$ & $0.0758(3)$ & $7.3(1)$ \\
\hline$C(25)$ & $0.7278(3)$ & $0.8347(2)$ & $0.2478(3)$ & $5.46(9)$ \\
\hline $\mathbf{H}(211)$ & $0.867(3)$ & $0.593(3)$ & $0.178(2)$ & $8(1)^{\star}$ \\
\hline$H(212)$ & $0.853(3)$ & $0.658(2)$ & $0.271(2)$ & $8(1)^{\star}$ \\
\hline $\mathbf{H}(213)$ & $0.796(3)$ & $0.573(2)$ & $0.246(2)$ & $7.3(9)^{\star}$ \\
\hline$H(221)$ & $0.631(3)$ & $0.531(2)$ & $-0.017(2)$ & $7.0(9)^{\star}$ \\
\hline$H(222)$ & $0.746(4)$ & $0.555(3)$ & $-0.012(3)$ & $12(1)^{\star}$ \\
\hline$H(223)$ & $0.702(4)$ & $0.497(3)$ & $0.076(3)$ & $13(1)^{\star}$ \\
\hline$H(231)$ & $0.448(3)$ & $0.757(2)$ & $-0.067(2)$ & $7.1(9)^{\star}$ \\
\hline$H(232)$ & $0.492(3)$ & $0.661(21)$ & $-0.095(3)$ & $8(1)^{\star}$ \\
\hline$H(233)$ & $0.548(3)$ & $0.726(3)$ & $-0.112(2)$ & $9(1)^{\star}$ \\
\hline$H(241)$ & $0.462(3)$ & $0.882(2)$ & $0.047(2)$ & $7.1(9)^{\star}$ \\
\hline$H(242)$ & $0.586(4)$ & $0.937(3)$ & $0.065(3)$ & $12(1)^{\star}$ \\
\hline$H(243)$ & $0.510(3)$ & $0.908(3)$ & $0.125(2)$ & $9(1)^{\star}$ \\
\hline $\mathbf{H}(251)$ & $0.771(3)$ & $0.810(2)$ & $0.297(2)$ & $6.5(9)^{\star}$ \\
\hline $\mathbf{H}(252)$ & $0.783(3)$ & $0.878(2)$ & $0.225(2)$ & $6.9(9)$ \\
\hline $\mathbf{H}(253)$ & $0.674(3)$ & $0.872(3)$ & $0.268(2)$ & $8(1)^{\star}$ \\
\hline
\end{tabular}

${ }^{a}$ Mit $\star$ versehene Atome wurden isotrop verfeinert. Der isotrope äquivalente thermische Parameter $B$ für anisotrop verfeinerte Atome ist definiert durch: $4 / 3\left[a^{2} B(1,1)+b^{2} B(2,2)+c^{2} B(3,3)+\right.$ $a b(\cos \gamma) B(1,2)+a c(\cos \beta) B(1,3)+b c(\cos \alpha) B(2,3)]$

Tabelle 2

Ausgewählte Bindungslängen $(\AA)$ und -winkel $\left({ }^{\circ}\right)$ für $\mathrm{CP}_{2}{ }^{\star} \mathrm{Rh}_{2}(\mathrm{CO})_{2} \mathrm{Se}$ (VIII)

\begin{tabular}{llll}
\hline $\mathrm{Rh}-\mathrm{Rh}^{\prime}$ & $2.755(1)$ & $\mathrm{Rh}-\mathrm{Se}-\mathrm{Rh}^{\prime}$ & $68.73(1)$ \\
$\mathrm{Rh}-\mathrm{Se}$ & $2.440(1)$ & $\mathrm{Rh}-\mathrm{Rh}^{\prime}-\mathrm{C}$ & $90.21(7)$ \\
$\mathrm{Rh}-\mathrm{C}$ & $1.834(2)$ & $\mathrm{Se}-\mathrm{Rh}-\mathrm{C}$ & $\mathbf{8 8 . 7 4 ( 7 )}$ \\
$\mathrm{Rh}-\mathrm{C}(11)$ & $2.274(2)$ & & \\
$\mathrm{Rh}-\mathrm{C}(12)$ & $2.243(2)$ & \\
$\mathrm{Rh}-\mathrm{C}(13)$ & $2.203(2)$ & \\
$\mathrm{Rh}-\mathrm{C}(14)$ & $2.229(2)$ & & \\
$\mathrm{Rh}-\mathrm{C}(15)$ & $2.302(2)$ & & \\
$\mathrm{C}-\mathrm{O}$ & $1.145(2)$ & & \\
\hline
\end{tabular}



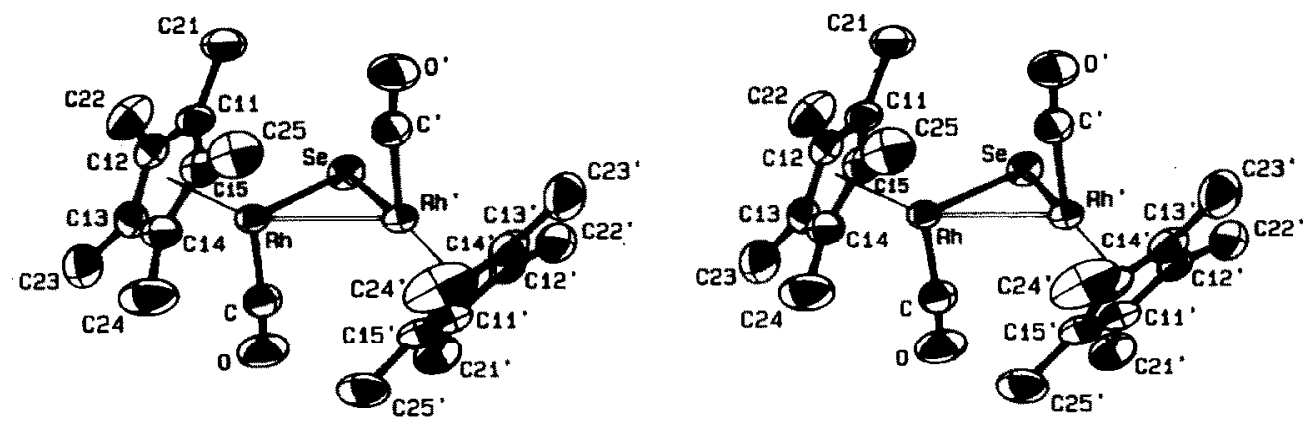

Fig. 1. Stereoansicht der Molekulstruktur von $\left(\mathrm{C}_{5} \mathrm{Me}_{5}\right)_{2} \mathrm{Rh}_{2}(\mathrm{CO})_{2} \mathrm{Se}$ (VIII). Die thermischen Schwingungsellipsoide entsprechen 50\% Aufenthaltswahrscheinlichkeit.

demonstriert wird. Die Tatsache, dass keine einfach verbrückten $\mathrm{Co}_{2} \mathrm{E}-\mathrm{Komplexe}$ in Substanz gefasst werden können, lässt vermuten, dass auch diese dem reaktiven Strukturtyp B angehören und sofort mit weiterem Chalkogen abreagieren.

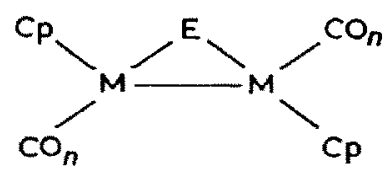

(A)

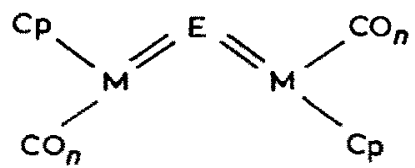

(B)

\begin{tabular}{ccc}
$M$ & Mn , Re & Co, Rn \\
\hline$n$ & 2 & 1
\end{tabular}

\section{Der Strukturtyp $\mathrm{Cp}_{2}^{\star} \mathrm{M}_{2}(\mathrm{CO})_{2} \mathrm{E}_{2}$}

Auf Grund der IR-Spektren ( $\nu(\mathrm{CO})$-Absorptionen im Bereich von $2000 \mathrm{~cm}^{-1}$, Tab. 3) enthalten die Komplexe IXa,b und XIa,b terminale CO-Liganden. Für die Stabilisierung der 16-e-Fragmente $\mathrm{Cp}^{\star} \mathrm{M}(\mathrm{CO})(\mathrm{M}=\mathrm{Co}, \mathrm{Rh})$ kommen somit nur 2-e- bzw. 4-e-Chalkogenidbrücken in Frage. Von den in Schema 3 aufgezeigten Möglichkeiten wurden mittels der vergleichbaren 16-e-Fragmente $\mathrm{C}_{5} \mathrm{R}_{5} \mathrm{M}(\mathrm{CO})_{2}$ $\left(\mathrm{R}=\mathrm{H}, \mathrm{CH}_{3} ; \mathrm{M}=\mathrm{Mn}, \mathrm{Re}\right.$ ) bisher $\eta^{1}-\mathrm{S}_{2}-[18]$ und $\eta^{1}, \eta^{2}-\mathrm{Te}_{2}-$ [19] Brücken ( $\mathrm{D}$ bzw. E) stabilisiert und auch strukturell charakterisiert. Eine nicht symmetrische Koordinationsform scheidet im vorliegenden Fall jedoch aus, da in IX und XI äquivalente $\mathrm{Cp}^{\star}$-Liganden vorliegen (s. ${ }^{1} \mathrm{H}$-NMR-Spektren, Tab. 3).<smiles></smiles><smiles>FPC[W]</smiles>

(C)

(D)<smiles>FN1C[AlH]1</smiles>

(E)

Schema 3. Mögliche Isomere für $\mathrm{L}_{2} \mathrm{M}_{2} \mathrm{E}_{2}-\mathrm{Komplexe}$, wobei $\mathrm{E}=\mathrm{S}, \mathrm{Se}, \mathrm{Te}\left(\mathrm{L}=\mathrm{Cp}{ }^{\star}(\mathrm{CO})_{2}: \mathrm{M}=\mathrm{Mn}, \mathrm{Re}\right.$; $\left.L=C_{p}^{\star}(C O): M=C o, R h\right)$. 
Tabelle 3

Spektroskopische Daten der Komplexe IV-XI

\begin{tabular}{lll}
\hline & $\begin{array}{l}\text { IR }\left(\mathrm{cm}^{-1}, \mathrm{KBr}\right) \\
\nu(\mathrm{CO})\end{array}$ & $\begin{array}{l}{ }^{1} \mathrm{H}_{-} \mathrm{NMR}^{b} \\
\left(\delta\left(\mathrm{CH}_{3}\right)\right)\end{array}$ \\
\hline IV & 1957 & $1.93^{c}$ \\
V & \multicolumn{1}{c}{$a$} & $1.85^{c}$ \\
VI & 1811 & $1.85,1.68^{c}$ \\
VII & - & $1.93^{d}$ \\
VIII & 1953 & $1.78^{d}$ \\
IXa & 1988 & $1.75^{e}$ \\
IXb & 2000 & $1.52^{c}$ \\
Xa & - & $1.66^{c}$ \\
Xb & - & $1.98^{c}$ \\
XIa & 1972 & $2.07^{c}$ \\
XIb & 1990 &
\end{tabular}

${ }^{a}$ Siehe Text. ${ }^{b} \mathrm{CDCl}_{3}$-Lösung, i-TMS. ${ }^{c}$ Varian EM-360L Spektrometer $\left(33^{\circ} \mathrm{C}\right) .{ }^{d}$ Gerät Bruker WM250 $\left(0^{\circ} \mathrm{C}\right) .{ }^{e} \mathrm{C}_{6} \mathrm{D}_{6}$-Lösung.

Die $\eta^{1}-S_{2}$-Form $\mathbf{D}$ enthält einen $\sigma$-Donor-Liganden. Eine derartige Koordinationsform konnte für Tellur bisher noch nicht nachgewiesen werden. Die S- und Se-Komplexe liessen sich bis jetzt nur durch Umsetzung hochreaktiver CpCarbonyl-Komplexe mit den entsprechenden Chalkogenen synthetisieren [20]. Prinzipiell denkbar wäre jedoch auch der Einschub von Chalkogen in die Metall-E-Bindung, wie der Aufbau einer $\mathrm{Cr}-\mathrm{S}_{2}-\mathrm{Cr}$-Einheit aus dem $\mathrm{Cr}-\mathrm{S}-\mathrm{Cr}$ Strukturelement und $S_{8}$ demonstriert [21]. Planare $M_{2} E_{2}$-Vierringgerüste (Form $C$ ) sind ausgesprochen häufig für $\mathrm{E}=\mathrm{S}$, selbst wenn nur Cyclopentadienylkomplexe berücksichtigt werden [22]. Weniger Beachtung fanden in diesem Zusammenhang bisher Selenkomplexe. Als einziger Tellurkomplex dieser Geometrie ist bisher [(t$\left.\left.\mathrm{BuC}_{5} \mathrm{H}_{4}\right)_{2} \mathrm{ZrTe}\right]_{2}$ strukturell charakterisiert worden [23]. Ein Hinweis auf mögliche syn/anti-Anordnung der $\mathrm{Cp}^{\star}$ - bzw. CO-Liganden ist lediglich dem ${ }^{1} \mathrm{H}-\mathrm{NMR}$ Spektrum von XIb zu entnehmen: ein bei $\delta=2.36 \mathrm{zu} 10 \%$ vorhandenes Resonanzsignal wird dem syn-Isomeren zugeschrieben. Diese Zuordnung basiert auf der Annahme einer stereoselektiven Öffnung des $\mathbf{M}_{2} \mathrm{E}$-Dreirings, in dem die antiAnordnung vorgegeben ist (siehe Röntgenstrukturanalyse von VIII), durch das neu eintretende Chalkogen.

\section{Die Molekülstruktur von $\mathrm{Cp}_{2}^{\star} \mathrm{Co}_{2} \mathrm{Se}_{5}$ (Xa)}

Einkristalle von $\mathrm{Xa}$ wurden aus Toluollösung gewonnen und einer Röntgenstrukturanalyse unterzogen. Atomkoordinaten und ausgewählte Bindungsparameter sind in den Tabellen 4 und 5 aufgelistet. Wie bereits in $\mathrm{Cp}^{\star}{ }_{2} \mathrm{Rh}_{2} \mathrm{Se}_{5}(\mathrm{Xb})$ [1], sind die beiden Co-Atome durch einen $\mathrm{Se}_{4}{ }^{2-}$ - und einen $\mathrm{Se}^{2-}$-Liganden verbrückt (Fig. 2). Der hierdurch gebildete, nahezu planare Pseudofünfring halbiert die Co-Co-Achse und steht auf dieser senkrecht. Da er als reiner $\sigma$-Donor sechs Elektronen pro $\mathrm{Co}^{\prime \prime \prime}$-Zentrum beisteuert, ist nach der Gesamtelektronenbilanz keine bindende $\mathbf{M}-\mathbf{M}$-Wechselwirkung zu erwarten. Dem entspricht der gefundene $\mathrm{Co}-\mathrm{Co}-\mathrm{Abstand}$ von $3.15 \AA$. Die Se-Se-Bindungen werden durch die Koordination praktisch nicht beeinflusst, ihre Werte liegen im Bereich der für die gängigen Selen-Modifikationen gefundenen Bingungslängen [24]. 
Tabelle 4

Atomkoordinaten von $\mathrm{Cp}_{2}^{\star} \mathrm{Co}_{2} \mathrm{Se}_{5}(\mathrm{Xa})$

\begin{tabular}{llcc}
\hline Atom & $x / a$ & $y / b$ & $z / c$ \\
\hline Co(1) & $-0.0734(2)$ & $-0.1719(5)$ & $0.1682(3)$ \\
$\operatorname{Se}(1)$ & $0.0000(0)$ & $0.3661(7)$ & $0.2500(0)$ \\
$\operatorname{Se}(2)$ & $-0.0351(2)$ & $-0.2703(4)$ & $0.3243(2)$ \\
$\operatorname{Se}(3)$ & $0.0264(2)$ & $0.4501(5)$ & $0.1928(3)$ \\
C(1) & $-0.136(1)$ & $-0.045(4)$ & $0.053(2)$ \\
C(2) & $-0.132(2)$ & $-0.219(4)$ & $0.036(2)$ \\
C(3) & $-0.167(1)$ & $-0.044(4)$ & $0.119(2)$ \\
C(4) & $-0.178(2)$ & $-0.213(5)$ & $0.129(3)$ \\
C(5) & $-0.160(2)$ & $-0.313(5)$ & $0.088(3)$ \\
C(6) & $-0.097(3)$ & $-0.175(8)$ & $-0.036(4)$ \\
C(7) & $-0.133(3)$ & $-0.442(8)$ & $0.011(4)$ \\
$C(8)$ & $-0.113(3)$ & $0.137(6)$ & $0.019(4)$ \\
$C(9)$ & $-0.215(3)$ & $-0.176(7)$ & $0.220(4)$ \\
$C(10)$ & $-0.183(3)$ & $0.130(8)$ & $0.175(4)$ \\
\hline
\end{tabular}

Tabelle 5

Ausgewählte Bindungslängen $(\AA)$ und -winkel $\left({ }^{\circ}\right)$ für $\mathrm{Cp}_{2}^{\star}{ }_{2} \mathrm{Co}_{2} \mathrm{Se}_{5}(\mathrm{Xa})$

\begin{tabular}{lllr}
\hline $\operatorname{Co}(1)-\operatorname{Se}(1)$ & $2.364(6)$ & $\operatorname{Co}(1)-\operatorname{Se}(1)-\operatorname{Co}(1 \mathrm{~A})$ & $83.6(3)$ \\
$\operatorname{Co}(1)-\operatorname{Se}(2)$ & $2.347(5)$ & $\operatorname{Co}(1)-\operatorname{Se}(2)-\operatorname{Co}(1 \mathrm{~A})$ & $84.4(2)$ \\
$\operatorname{Co}(2)-\operatorname{Se}(2 \mathrm{~A})$ & $2.348(6)$ & $\operatorname{Co}(1)-\operatorname{Se}(2)-\operatorname{Se}(3)$ & $104.4(2)$ \\
$\operatorname{Co}(1 \mathrm{~A})-\operatorname{Se}(1)$ & $2.365(6)$ & $\operatorname{Se}(2)-\operatorname{Se}(3)-\operatorname{Se}(3 \mathrm{~A})$ & $98.7(1)$ \\
$\operatorname{Co}(1 \mathrm{~A})-\operatorname{Se}(2)$ & $2.348(6)$ & $\operatorname{Se}(1)-\operatorname{Se}(2)-\operatorname{Se}(2 \mathrm{~A})$ & $59.5(1)$ \\
$\operatorname{Se}(2)-\operatorname{Se}(3)$ & $2.390(5)$ & $\operatorname{Se}(1)-\operatorname{Co}(1)-\operatorname{Se}(2)$ & $79.4(2)$ \\
$\operatorname{Se}(3)-\operatorname{Se}(3 \mathrm{~A})$ & $2.334(9)$ & & \\
\hline
\end{tabular}

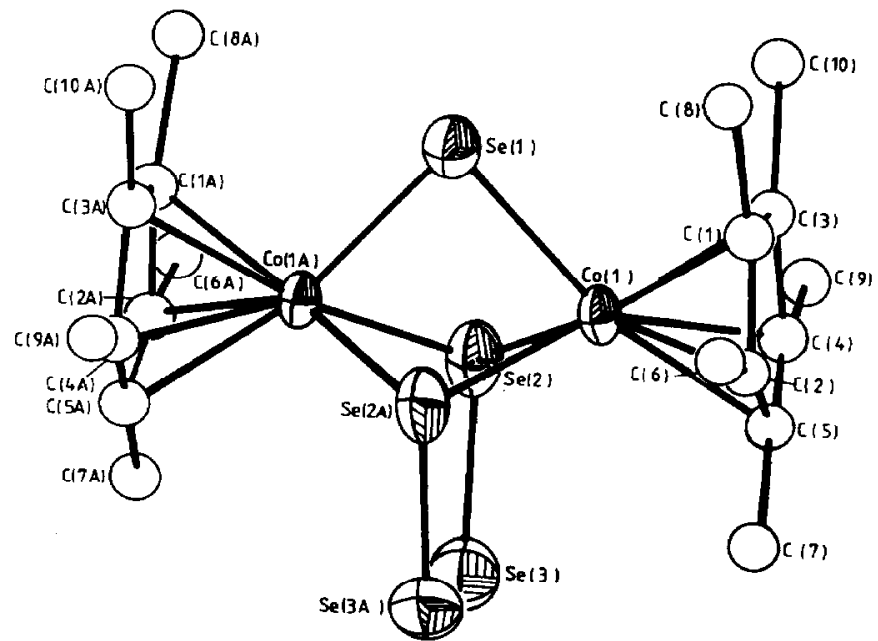

Fig. 2. Molekülstruktur von $\left(\mathrm{C}_{5} \mathrm{Me}_{5}\right)_{2} \mathrm{Co}_{2} \mathrm{Se}_{5}$ (Xa) (ORTEP-Zeichnung). 


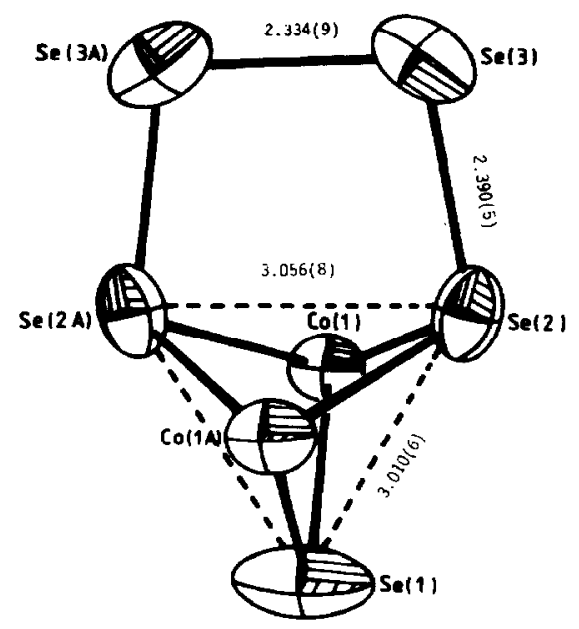

Fig. 3. Mulekülansicht von Xa entlang der $\mathrm{Co}-\mathrm{Co}$-Achse mil Abständen in $\AA$ (die $\mathrm{C}_{5} \mathrm{Me}_{5}$-Liganden sind weggelassen).

Ein Blick entlang der Co-Co-Achse (Fig. 3) zeigt, dass $\mathrm{Se}(1), \operatorname{Se}(2)$ und $\mathrm{Se}(2 \mathrm{~A})$ ein praktisch gleichseitiges Dreieck bilden mit Abständen zwischen 3.01 und $3.06 \AA$. Diese Werte liegen weit unter der Summe der Van-der-Waals-Radien zweier SeAtome (3.80 ̊ [25]) und könnten somit einen Anhaltspunkt für die Erklärung der in Komplexen des Typs X bevorzugten planaren Pseudofünfringgeometrie bieten. Es ist bekannt, dass Wechselwirkungen dieser Grössenordnung z. B. zur Dimerisierung zweier $\left[\mathrm{W}(\mathrm{CO})_{5}\left(\eta^{2}-\mathrm{Se}_{2}\right)\right]^{4}$-Moleküle in ein Molekül mit $\mathrm{Se}_{4}{ }^{2}{ }^{\prime}$-Brücke ausreichen [26]. Ein ähnlicher Effekt dürfte, wenn auch in deutlich abgeschwächter Form, in den Molekülgittern von Xa,b wirksam sein. Wie in Fig. 4 am Beispiel der im Vergleich zu Cp${ }_{2}{ }_{2} \mathrm{Co}_{2} \mathrm{Se}_{5}$ dichter gepackten Elementarzelle von $\mathrm{CP}^{\star}{ }_{2} \mathrm{Rh}_{2} \mathrm{Se}_{5}$ gezeigt, führen intermolekulare Wechselwirkungen $\left(d_{\mathrm{Se}-\mathrm{Se}} 3.50 \AA\right)$ zwischen dem $\mathrm{Se}^{2-}$ -

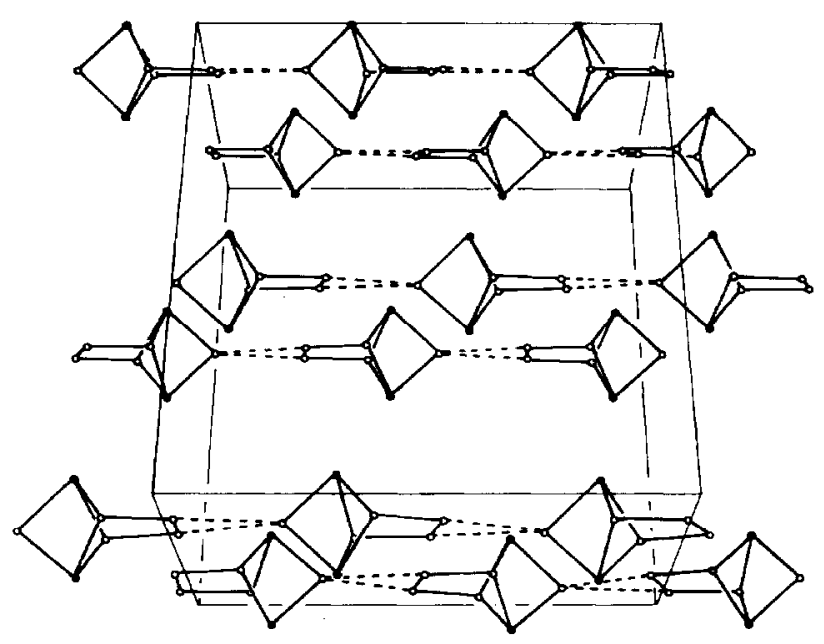

Fig. 4. Ansicht der Elementarzelle von $\left(\mathrm{C}_{5} \mathrm{Me}_{5}\right)_{2} \mathrm{Rh}_{2} \mathrm{Se}_{5}[1]\left(\mathrm{O}=\mathrm{Se}-\mathrm{Atome}, \bullet=\mathrm{Rh}\right.$-Atome; die $\mathrm{C}_{5} \mathrm{Me}_{5}-$ Liganden sind aus Gründen der Übersichtlichkeit weggelassen). 
Liganden des einen Moleküls mit dem $\mathrm{Se}_{4}{ }^{2-}$-Liganden eines weiteren Moleküls zu einem für Cyclopentadienylkomplexe recht ungewöhnlichen eindimensionalen Aufbau: Sämtliche Se-Atome liegen in einer Ebene, die durch die hierzu parallelen $\mathrm{Cp}{ }^{\star} \mathrm{Rh}$-Fragmente zu einer kompakten Schicht ergänzt werden.

\section{Diskussion}

Ein bedeutsamer Unterschied gegenüber den $\mathbf{M}$-M-Dreifachbindungssystemen der Komplexe $\mathrm{Cp}_{2}{ }_{2} \mathrm{M}_{2}(\mathrm{CO})_{4}(\mathrm{M}=\mathrm{Cr}, \mathrm{Mo}, \mathrm{W})$ [27] ist die Fähigkeit der $\mathrm{M}-\mathrm{M}-$ Doppelbindung in $\left[\mathrm{Cp}^{\star} \mathrm{M}(\mathrm{CO})\right]_{2}(\mathrm{M}=\mathrm{Co}, \mathrm{Rh})$ zur Addition von ein bzw. zwei Chalkogenatomen , unter Ausbildung relativ stabiler Zwischenprodukte. Da sich diese in den meisten Fällen mit überschüssigem Chalkogen in die CO-freien Endprodukte überführen lassen (IV $\rightarrow$ VII; VIII bzw. IXa,b $\rightarrow \mathrm{Xa}, \mathrm{b}$ ) sollten sie auch den Schlüssel zu deren Aufbau beinhalten. Aus diesem Grund wurde die Synthese von Zweikernkomplexen mit gemischten Chalkogenidbrücken versucht. Als Modellsubstanzen wurden hierzu $\mathrm{Cp}_{2}^{\star} \mathrm{Rh}_{2}(\mathrm{CO})_{2} \mathrm{~S}(\mathrm{IV})$ und $\mathrm{Cp}_{2}^{\star} \mathrm{Rh}_{2}(\mathrm{CO})_{2} \mathrm{Se}$ (VIII) mit einem Äquivalent Selen bzw. Schwefel umgesetzt. Anstelle der erhofften Verbindung $\mathrm{Cp}^{\star}{ }_{2} \mathrm{Rh}_{2}(\mathrm{CO})_{2}(\mu-\mathrm{S})(\mu-\mathrm{Se})$ wurde entweder keine Reaktion (im Fall von IV) oder die Bildung von $\mathrm{CO}$ - und Se-freiem (!) $\mathrm{Cp}_{2}^{\star}{ }_{2} \mathrm{Rh}_{2} \mathrm{~S}_{8}$ (VII) zusammen mit unverbrauchtem VIII beobachtet. Somit sind überraschenderweise nicht nur die CO-Liganden als substitutionslabil anzunehmen, sondern auch die Chalkogenidbrücken.

Aufgrund des derzeitigen Kenntnisstandes bieten sich für den Aufbau der hier berichteten Polychalkogenidstrukturen eine ganze Reihe mechanistischer Überlegungen an. In allen Fällen sollte zunächst ein Angriff des als nukleophil anzunehmenden Metallzentrums am Chalkogenring bzw. an der -kette deren Abbau zu Monochalkogenidliganden bewirken, die sich als Brücken in den Mono- bzw. Diaddukten IV, V, VIII, IX und XI wiederfinden. Dieses Reaktionsschema liegt in den meisten Fällen dem Schwefelabbau durch Übergangsmetallkomplexe zugrunde [20]. In der unterschiedlichen Stabilität der Addukte ist der Einfluss des Zentralmetalls deutlich spürbar, aber auch in der Reaktivität der eingesetzten Chalkogene ergeben sich deutliche Abstufungen ( $\mathrm{S}>\mathrm{Se}>\mathrm{Te}$ ).

Durch oxidative Addition von Schwefel oder Selen (Tellur scheidet aufgrund mangelhafter Reaktivität bereits aus) an die Metallzentren dieser Zwischenstufenbzw. -produkte werden nunmehr $\mathrm{ME}_{4}$-Chelate gebildet. Als einzige Ausnahme geht der $\mathrm{S}_{8}$-Abbau im $\mathrm{Co} / \mathrm{S}$-System bis zu $\mathrm{S}_{2}$-Einheiten weiter. Dieser Vorgang kann unterstützt werden durch die Fähigkeit der CO-Brücken zu ihrer reversiblen Öffnung $(\mathrm{II} \rightarrow$ IV $\rightarrow$ VI) sowie durch die Präsenz substitutionslabiler $\mathrm{CO}$ - und $\mu$-E-Liganden. Auch hier macht sich der Einfluss des Chalkogens bemerkbar, denn ein zu VI analoger Se-Komplex, der zwei $\mathrm{MSe}_{4}$-Einheiten enthalten würden, scheint auf Grund sterischer oder kinetischer Argumente nicht begünstigt. Diese Überlegungen sind jedoch als stark vereinfachend anzunehmen, denn Tieftemperatur-IR-Spektren machen zumindest im Rh/S-System die Beteiligung noch weiterer Zwischenstufen sehr wahrscheinlich.

Alternativ hierzu lassen sich jedoch auch Beweise für den stufenweise Aufbau der Polychalkogenidliganden aus reaktiven Einzelbausteinen aufführen. So "zcrsetzt" sich z. B. die $\mathrm{CHCl}_{3}$-Lösung von $\mathrm{Cp}_{2}^{\star} \mathrm{Co}_{2}(\mathrm{CO})_{2} \mathrm{Se}_{2}$ zu einem Gemisch, aus dem sich $\mathrm{Cp}^{\star}{ }_{2} \mathrm{Co}_{2} \mathrm{Se}_{5}$ isolieren lässt, und Spuren $\mathrm{H}_{2} \mathrm{Se}$, was die Entstehung von reakti- 
vem Se voraussetzt. Ein Pendant findet dieser Vorgang im Aufbau einer $\mathrm{Se}_{5}{ }^{2-}$-Brïcke in $\mathrm{V}_{2} \mathrm{Se}_{13}{ }^{2-}$ aus diskreten $\mathrm{Se}^{2-}$-Ionen [28]. Im Normalfall werden Polychalkogenidliganden als Zwischenstufen bei der Bildung von Mono- und Dichalkogenidkomplexen angenommen [29]. Die Tatsache, dass sich $\mathrm{Cp}^{\star}{ }_{2} \mathrm{Rh}_{2} \mathrm{~S}_{8}$ und $\mathrm{Cp}_{2}^{\star} \mathrm{Co}_{2} \mathrm{Se}_{5}$ selbst bei $100^{\circ} \mathrm{C}$ und in Gegenwart von $\mathrm{PPh}_{3}$ einer Degradierung ihrer relativ hohen Anzahl von E-E-Bindungen widersetzen, zeugt von einer überraschenden thermischen Stabilität dieser Komplexe $[29,30 *]$.

Weitere Arbeiten zum Verständnis des Bildungsmechanismus der hier vorgestellten neuartigen Polychalkogenidstrukturen sind geplant, z. B. der Einfluss der Substituenten am Cp-Liganden. Da nämlich in $\mathrm{C}_{5} \mathrm{H}_{5} \mathrm{MS}_{5}(\mathrm{M}=\mathrm{Co}$ [31], $\mathrm{Rh}$ [32] $)$ Sechsringchelate vorliegen, darf angenommen werden, dass wie in $\mathrm{Cp}_{2} \mathrm{TiS}_{n}$ Komplexen die Anzahl $n$ der Ringglieder vom sterischen Bedarf des Cp-Restes abhängt [29]. Weiteres Interesse erwecken die Komplexe Xa,b durch ihren Bezug zu Festkörperstrukturen: Dazu trägt neben der eindimensionalen Verknüpfung im Kristallgitter die $\ddot{A} h n l i c h k e i t$ des $\eta^{3}-\mathrm{Se}_{5}$-Liganden mit den $\mathrm{Se}_{5}$-Einheiten in $\mathrm{Nb}_{2} \mathrm{Se}_{9}$ bei, die $\mathrm{Nb}_{2} \mathrm{Se}_{4}$-Oktaeder verbrücken [33].

\section{Experimenteller Teil}

Alle Arbeiten wurden unter Luftausschluss und unter Verwendung von trockenen, $\mathrm{N}_{2}$-gesättigten Lösungsmitteln durchgeführt. Bei dem im folgenden verwendeten $\mathrm{Al}_{2} \mathrm{O}_{3}$ handelt es sich ausschliesslich um ein Produkt der Fa. Merck (Aktivität II-III), das möglichst schonend unter $\mathrm{N}_{2}$ gesetzt wurde. Vorsicht: Alle Ansätze sollten in einem gut ziehenden Abzug durchgeführt werden, da gelegentlich Spuren des hochtoxischen Selenwasserstoffs auftreten! Bei allen Versuchen wurden graues Selen (EGA-Chemie, 99.5\% Reinheit) und Tellur (Merck, 99\%) in handelsüblicher Qualität verwendet. Der Einsatz des reaktiveren roten, amorphen Selens [34] führt in der Regel zu gleicher Produktverteilung bei Verkürzung der Reaktionszeiten. Die Komplexe I [35], II [13] und IV [8] wurden nach Literaturangaben dargestellt. Zur Umkristallisation empfiehlt sich in allen Fällen eine Filtration der gesättigten Lösungen über wenig $\mathrm{Al}_{2} \mathrm{O}_{3}$. Elementaranalysen, Ausbeuten und weitere Eigenschaften sind in Tab. 6 zusammengefasst.

\section{Darstellung von $\mathrm{Cp}_{2}^{\star}{ }_{2} \mathrm{Rh}_{2}(\mathrm{CO})_{2} \mathrm{~S}(\mathrm{IV})$}

Die dunkelblaue Lösung von $210 \mathrm{mg}(0.39 \mathrm{mmol})$ II und $13 \mathrm{mg}(0.05 \mathrm{mmol}) \mathrm{S}_{8}$ in $50 \mathrm{ml}$ THF wird $15 \mathrm{~min}$ bei $20^{\circ} \mathrm{C}$ gerührt, wobei sich die Farbe nach braunrot ändert. Nach Entfernen des Lösungsmittels nimmt man den Rückstand in $10 \mathrm{ml}$ Toluol auf und chromatographiert an $\mathrm{Al}_{2} \mathrm{O}_{3}$ (Säule $15 \times 3 \mathrm{~cm}$ ). Mit Toluol eluiert man zunächst nicht umgesetztes II, gefolgt von Spuren einer roten, noch nicht identifizierten Verbindung. Weitere braune und grüne Verunreinigungen werden mit $\mathrm{Et}_{2} \mathrm{O}$ eluiert, ehe man den gewünschten Komplex IV als breite rotviolette Zone isolieren kann. Durch Umkristallisation aus Toluol/Pentan $(4 / 1)$ erhält man schwarzrote Kristalle.

\section{Darstellung der Komplexe VI und VII}

Die dunkelblaue Lösung von $200 \mathrm{mg}(0.38 \mathrm{mmol})$ II und $106 \mathrm{mg}(0.41 \mathrm{mmol}) \mathrm{S}_{8}$ in $100 \mathrm{ml}$ THF wird $15 \mathrm{~min}$ bei $-25^{\circ} \mathrm{C}$ gerührt. Die nunmehr rotbraune Lösung wird noch kalt über $\mathrm{Al}_{2} \mathrm{O}_{3}$ filtriert. Nach dem Entfernen des THF wird der 
Tabelle 6

Ausgewählte Eigenschaften, Ausbeuten und Analysenwerte der Komplexe IV, VII, IX-XI

\begin{tabular}{lllllll}
\hline Summenformel & Farbe & $\begin{array}{l}\text { Ausbeute } \\
(\%)\end{array}$ & $\begin{array}{l}\text { Molgew. }^{a} \\
\text { (Gef. (ber.)) }\end{array}$ & \multicolumn{3}{c}{ Analysen (Gef. (ber.) (\%)) } \\
\cline { 5 - 7 } & & & & $\mathrm{C}$ & $\mathrm{H}$ & $\mathrm{E}$ \\
\hline $\mathrm{C}_{22} \mathrm{H}_{30} \mathrm{Rh}_{2} \mathrm{O}_{2} \mathrm{~S}$ & rotviolett & $40-48$ & 564 & 47.00 & 5.49 & \\
$(\mathrm{IV})$ & & & 564.4 & 46.82 & 5.36 & \\
$\mathrm{C}_{20} \mathrm{H}_{30} \mathrm{Rh}_{2} \mathrm{~S}_{8}$ & rotbraun & 69 & $b$ & 33.42 & 4.15 & $36.03(\mathrm{E}=\mathrm{S})$ \\
$(\mathrm{VII})$ & & & 732.8 & 32.78 & 4.18 & 35.00 \\
$\mathrm{C}_{22} \mathrm{H}_{30} \mathrm{Co}_{2} \mathrm{O}_{2} \mathrm{Se}_{2}$ & dunkelbraun & 25 & $576^{c}$ & 43.36 & 5.00 & $26.60(\mathrm{E}=\mathrm{Se})$ \\
$(\mathrm{IXa})$ & & & 602 & 43.87 & 5.02 & 26.22 \\
$\mathrm{C}_{22} \mathrm{H}_{30} \mathrm{Rh}_{2} \mathrm{O}_{2} \mathrm{Se}_{2}$ & dunkelbraun & 60 & $d$ & 38.53 & 4.49 & $23.20(\mathrm{E}=\mathrm{Se})$ \\
$(\mathrm{IXb})$ & & & 690.2 & 38.28 & 4.38 & 22.88 \\
$\mathrm{C}_{20} \mathrm{H}_{30} \mathrm{Co}_{2} \mathrm{Se}_{5}$ & violettbraun & 80 & $788^{e}$ & 30.67 & 3.78 & $50.60(\mathrm{E}=\mathrm{Se})$ \\
$(\mathrm{Xa})$ & & & 783.1 & 30.67 & 3.87 & 50.41 \\
$\mathrm{C}_{20} \mathrm{H}_{30} \mathrm{Rh}_{2} \mathrm{Se}_{5}$ & dunkelgrün & 19 & $876^{e}$ & 27.99 & 3.60 & \\
$(\mathrm{Xb})$ & & & 871.1 & 27.57 & 3.47 & \\
$\mathrm{C}_{22} \mathrm{H}_{30} \mathrm{Co}_{2} \mathrm{O}_{2} \mathrm{Te}_{2}$ & dunkelbraun & 88 & $699^{f}$ & 37.48 & 4.26 & $38.05(\mathrm{E}=\mathrm{Te})$ \\
$(\mathrm{XIa})$ & & & 699.5 & 37.77 & 4.32 & 36.48 \\
$\mathrm{C}_{22} \mathrm{H}_{30} \mathrm{Rh}_{2} \mathrm{O}_{2} \mathrm{Te}_{2}$ & grünbraun & 34 & $787^{f}$ & 33.19 & 3.84 & \\
$(\mathrm{XIb})$ & & & 787.5 & 33.55 & 3.84 & \\
\hline
\end{tabular}

${ }^{a}$ Felddesorptionsmassenspektren (Gerät Varian 311A, Toluollösung). ${ }^{b}$ Fadenheizung bis $6 \mathrm{~mA}:$ [M $\mathrm{S}]^{+\cdot}\left(m / e\right.$ 700); $>10 \mathrm{~mA}:[M-2 \mathrm{~S}]^{+\cdot}\left(m / e\right.$ 668). ${ }^{c}[M-\mathrm{CO}]^{+*}:{ }^{d}$ Als einziger Peak wird $m / e=1268$ beobachtet, der rein rechnerisch einer Zusammensetzung $\left(\mathrm{C}_{5} \mathrm{Me}_{5}\right)_{4} \mathrm{Rh}_{4} \mathrm{Se}_{4}$ entspricht. ${ }^{e} \mathrm{Bzgl} .{ }^{80} \mathrm{Se}$. ${ }^{f}$ Bzgl. ${ }^{128} \mathrm{Te}$.

Rückstand in $20 \mathrm{ml}$ Toluol gelöst und an $\mathrm{Al}_{2} \mathrm{O}_{3}$ (Säule $23 \times 4 \mathrm{~cm}$ ) chromatographiert (Raumtemperatur). Mit Toluol/Aceton (10/1) eluiert man zunächst $72 \mathrm{mg}(25 \%)$ rotbraunes $\mathrm{Cp}^{\star}{ }_{2} \mathrm{Rh}_{2}(\mathrm{CO}) \mathrm{S}_{8}$ (VI) und dann $74 \mathrm{mg}(26 \%)$ braunes $\mathrm{Cp}^{\star}{ }_{2} \mathrm{Rh}_{2} \mathrm{~S}_{8}$ (VII). Komplex VII lässt sich in besseren Ausbeuten durch $18 \mathrm{~h}$ Rühren von $\mathrm{S}_{8}$ und II im wie oben angegebenen Verhältnis bei Raumtemperatur darstellen. Dunkelbraune Prismen von VII lassen sich durch Umkristallisation aus Toluol gewinnen.

Darstellung von $\mathrm{Cp}_{2}{ }_{2} \mathrm{Co}_{2}(\mathrm{CO})_{2} \mathrm{Se}_{2}(\mathrm{IXa})$

Ein Gemisch aus $590 \mathrm{mg}(1.33 \mathrm{mmol}) \mathrm{I}, 580 \mathrm{mg}(7.34 \mathrm{mmol})$ grauem Selen und $50 \mathrm{ml}$ THF wird $30 \mathrm{~min}$ bei $0^{\circ} \mathrm{C}$ gerührt. Nicht umgesetztes Selen wird durch Filtration der kalten Lösung über $\mathrm{Al}_{2} \mathrm{O}_{3}$ entfernt. Das Solvens wird in der Kälte abgezogen. Der graubraune Rückstand wird in $20 \mathrm{ml} \mathrm{CH}_{2} \mathrm{Cl}_{2}\left(0^{\circ} \mathrm{C}\right)$ aufgenommen und über wenig $\mathrm{Al}_{2} \mathrm{O}_{3}$ filtriert, wobei mit $10 \mathrm{ml} \mathrm{CH}_{2} \mathrm{Cl}_{2}$ nachgewaschen wird. Nach Konzentrieren auf ca. $15 \mathrm{ml}$ und Abkühlen auf $-25^{\circ} \mathrm{C}$ fallen $200 \mathrm{mg}(25 \%)$ dunkelbraune Kristalle aus.

Darstellung von $\mathrm{Cp}^{\star}{ }_{2} \mathrm{Rh}_{2}(\mathrm{CO})_{2} \mathrm{Se}_{2}(\mathrm{IXb})$

Ein Gemisch aus $260 \mathrm{mg}(0.49 \mathrm{mmol}) \mathrm{II}, 80 \mathrm{mg}(1.01 \mathrm{mmol})$ rotem Selen und 40 $\mathrm{ml}$ THF werden zunächst $10 \mathrm{~min}$ bei Raumtemperatur (die hierbei beobachtete Farbänderung von blau nach rot entspricht der Bildung des Monoaddukts VIII [8]) und dann 60 min bei $50^{\circ} \mathrm{C}$ gerührt. Sodann wird die Reaktionslösung über $\mathrm{Al}_{2} \mathrm{O}_{3}$ filtriert und nach dem Entfernen des Lösungsmittels einer Chromatographie an $\mathrm{Al}_{2} \mathrm{O}_{3}$ (Säule $18 \times 3 \mathrm{~cm}$ ) unterworfen. Mit Toluol $/ \mathrm{Et}_{2} \mathrm{O}(100 / 1)$ eluiert man 
zunächst Spuren eines rotbraunen, $\mathrm{CO}$-freien Komplexes, während IXb mit Toluol/ $\mathrm{Et}_{2} \mathrm{O}(10 / 1)$ als dunkelbraune Zone isoliert wird (201 $\left.\mathrm{mg}, 60 \%\right)$. Eine weitere, rotviolette Zone kann mit Toluol/ $\mathrm{Et}_{2} \mathrm{O}(1 / 3)$ eluiert werden und liefert VIII in geringen Mengen.

Darstellung von $C p^{\star}{ }_{2} M_{2} S e_{5}(M=C o: X a ; M=R h: X b)$

Eine Mischung aus $440 \mathrm{mg}(5.60 \mathrm{mmol})$ grauem Selen, $0.7 \mathrm{mmol}$ I oder II und $100 \mathrm{ml}$ Toluol wird $18 \mathrm{~h}$ bei Raumtemperatur gerührt. Nach Konzentrieren auf 20 $\mathrm{ml}$ wird an $\mathrm{Al}_{2} \mathrm{O}_{3}$ (Säule $20 \times 3 \mathrm{~cm}$ ) chromatographiert. Während $\mathrm{Xa}$ mit Toluol als dunkelviolette Zone eluiert wird, wird für das grüne $\mathrm{Xb}$ Toluol/ $\mathrm{Et}_{2} \mathrm{O}(20 / 1)$ benötigt. Beide Produkte werden vorteilhaft aus Toluol umkristallisiert.

\section{Darstellung von $\mathrm{Cp}_{2}{ }_{2} \mathrm{Co}_{2}(\mathrm{CO})_{2} \mathrm{Te}_{2}(\mathrm{XIa})$}

Ein Gemisch aus $380 \mathrm{mg}(0.86 \mathrm{mmol}) \mathrm{I}, 874 \mathrm{mg}(6.88 \mathrm{mmol})$ Tellur und $100 \mathrm{ml}$ Toluol wird $18 \mathrm{~h}$ bei $40^{\circ} \mathrm{C}$ gerührt. Die nunmehr braune Lösung wird über $\mathrm{Al}_{2} \mathrm{O}_{3}$ filtriert, wobei mit Toluol bis fast zur Farblosigkeit des Filtrats nachgewaschen wird. Das nach dem Abziehen des Lösungsmittels verbleibende dunkelbraune Pulver (530 $\mathrm{mg}, 88 \%$ ) wird aus Toluol bei $-25^{\circ} \mathrm{C}$ umkristallisiert.

Darstellung von $C p^{\star}{ }_{2} R h_{2}(C O)_{2} T e_{2}(X I b)$

Ein Gemisch aus $200 \mathrm{mg}(0.42 \mathrm{mmol}) \mathrm{II}, 430 \mathrm{mg}$ (3.36 mmol) Tellur und $100 \mathrm{ml}$ Toluol wird $18 \mathrm{~h}$ bei $60^{\circ} \mathrm{C}$ gerührt. Die grünbraune Lösung wird über $\mathrm{Al}_{2} \mathrm{O}_{3}$ filtriert, um überschüssiges Te zu entfernen. Nach Konzentration des Filtrats auf 10 $\mathrm{ml}$ chromatographiert man an $\mathrm{Al}_{2} \mathrm{O}_{3}$ (Säule $30 \times 4 \mathrm{~cm}$ ), wobei das Produkt mit Toluol als breite grünbraune Zone eluiert wird. Durch Umkristallisation aus Toluol/Pentan $(2 / 1)$ bei $-25^{\circ} \mathrm{C}$ erhält man feine grüne Kristalle.

Röntgenstrukturanalyse von $\mathrm{Cp}^{\star}{ }_{2} \mathrm{Rh}_{2}(\mathrm{CO})_{2} \mathrm{Se}(\mathrm{VIII})\left[36^{*}\right]$

Kleine schwarze, im Durchlicht auberginfarbene Kristalle mit unregelmässigen Kanten und Flächen (umkristallisiert aus $\mathrm{CH}_{2} \mathrm{Cl}_{2}$ ); monoklin, $\mathrm{C2} / c$; Zellkonstanten: $a$ 11.477(1), $b$ 13.989(2), $c$ 14.452(3) $\AA, \beta$ 98.68(1) ${ }^{\circ} ; \quad V 2294 \AA^{3} ; Z=4$; $d$ (röntg) $1.770 \mathrm{~g} \mathrm{~cm}^{-3} ; \mu 30.0 \mathrm{~cm}^{-1}$; Enraf-Nonius CAD4; $\lambda 0.71073 \AA$ (Mo-K $\alpha_{\alpha^{-}}$ Strahlung, Graphitmonochromator); Messbereich: $2^{\circ} \leq \theta \leq 25^{\circ}$; Anzahl der gemessenen Reflexe: 4163; unabhängige Reflexe: 1996, davon 231 mit $I<1.0 \sigma(I)$; Parameter full matrix verfeinert; $R=\Sigma|| F_{0}|-| F_{\mathrm{c}} \| / \Sigma\left|F_{0}\right|=0.021 ; \quad R_{\mathrm{w}}=$ $\left[\Sigma w\left(\left|F_{0}\right|-\left|F_{\mathrm{c}}\right|\right)^{2} / \Sigma w\left|F_{0}\right|^{2}\right]^{1 / 2}=0.020, \quad G O F=\left[\Sigma w\left(\left|F_{0}\right|-\left|F_{\mathrm{c}}\right|\right)^{2} /(N O-\right.$ $N V)]^{1 / 2}=2.394$ mit $w=1 / \sigma^{2}\left(F_{0}\right)$; Strukturlösung nach Patterson-Methode und sich daran anschliessenden Differenz-Fourier-Synthesen; empirische Absorptionskorrektur, $\mu 30.0 \mathrm{~cm}^{-1}$; keine Zersetzung; alle Nichtwasserstoffatome anisotrope Temperaturfaktoren; Wasserstofflagen sind sukzessive aus den Differenzfouriersynthesen entnommen und mit isotropen Temperaturfaktoren verfeinert; anomale Dispersion berücksichtigt; shift/err $<0.00$ im letzten Verfeinerungszyklus; Restelektronendichte $+0.48 \mathrm{e} / \AA^{3}$.

Röntgenstrukturanalyse von $\mathrm{Cp}_{2}^{\star} \mathrm{Co}_{2} \mathrm{Se}_{5}(\mathrm{Xa})\left[36^{*}\right]$

Kristallgrösse: $0.3 \times 0.2 \times 0.4 \mathrm{~mm}$; monoklin, $C 2 / c$; Zellkonstanten: $a$ 20.58(1), $b$ 8.45(3), c 15.01(3) $\AA, \beta$ 110.55(3) ${ }^{\circ} ; V 2446 \AA^{3} ; Z=4$; $d$ (röntg) $2.1 \mathrm{~g} \mathrm{~cm}^{-3}$; $\mu$ $85.65 \mathrm{~cm}^{-1}$; Syntex P3-Vierkreisdiffraktometer; Mo- $K_{\alpha}-$ Strahlung; Messbereich $3^{\circ}$ 
$\leq 2 \theta \leq 50^{\circ} ; 1012$ vermessene Reflexe, davon 989 voneinander unabhängige Reflexe; Patterson- (Co, Se) und Fourier-Methoden, Verfeinerung bis $R$ (isotrop) $=$ $0.13 ; R$ (anisotrop) $=0.08 ; R_{\mathrm{w}}=0.08$.

\section{Literatur}

1 H. Brunner, W. Meier, B. Nuber, J. Wachter und M.L. Ziegler, Angew. Chem., 98 (1986) 907; Angew. Chem. Int. Ed. Engl., 25 (1986) 908.

2 H. Brunner, E. Guggolz, J. Wachter und M.L. Ziegler, J. Am. Chem. Soc., 104 (1982) 1765.

3 H. Brunner, W. Meier, J. Wachter, E. Guggolz, T. Zahn und M.L. Ziegler, Organometallics, 1 (1982) 1107.

4 H. Brunner, J. Wachter und H. Wintergerst, J. Organomet. Chem., 235 (1985) 77.

5 H. Brunner, N. Janietz, W. Meier, B. Nuber, J. Wachter und M.L. Ziegler, Angew. Chem., im Druck.

6 H. Brunner, N. Janietz, W. Meier, G. Sergeson, J. Wachter, T. Zahn und M.L. Ziegler, Angew. Chem., 97 (1985) 1056; Angew. Chem. Int. Ed. Engl., 24 (1985) 1060.

7 M. Smischek, Diplomarbeit, Universität Regensburg 1981.

8 W.A. Hetrmann, J. Organomet. Chem., 250 (1983) 319.

9 W.A. Herrmann, C. Bauer und J. Weichmann, Chem. Ber., 117 (1984) 1271; W.A. Herrmann und J. Weichmann, Organomet. Synth., 3 (1986) 287.

10 W.A. Herrmann, Angew. Chem., 98 (1986) 57; Angew. Chem. Int. Ed. Engl., 25 (1986) 56.

11 P. Granger, B. Gautheron, G. Tainturier und S. Pouly, Org. Magn. Res., 22 (1984) 701.

12 Eine Zusammenfassung der derzeit zugänglichen ${ }^{77}$ Se-Resonanzen in Übergangsmetallkomplexen bietet die Dissertation von H.-J. Kneuper, Technische Universität München, 1986.

13 W.A. Herrmann, J. Plank, C. Bauer, M.L. Ziegler, E. Guggolz und R. Alt, Z. Anorg. Allg. Chem., 487 (1982) 85.

14 W.A. Herrmann, J. Plank, M.L. Ziegler und P. Wülknitz, Chem. Ber., 114 (1981) 716.

15 C. Bianchini, C. Mealli, A. Meli und M. Sabat, J. Am. Chem. Soc., 107 (1985) 5317.

16 Obwohl von diesem Komplex noch keine Röntgenstrukturanalyse vorliegt, kann auf seine Struktur aus dem S-Oxid rückgeschlossen werden: I.-P Lorenz, J. Messelhäuser, W. Hiller und K. Haug, Angew. Chem., 97 (1985) 234; Angew. Chem. Int. Ed. Engl., 24 (1985) 228; I.-P. Lorenz, J. Messelhäuser, W. Hiller und M. Conrad, J. Organomet. Chem., 316 (1986) 121.

17 M. Herberhold, D. Reiner, K. Ackermann, U. Thewalt und T. Debaerdemaeker, Z. Naturforsch. B, 39 (1984) 1199.

18 M. Herberhold, D. Reiner, B. Zimmer-Gasser und U. Schubert, Z. Naturforsch. B, 35 (1980) 1281.

19 W.A. Herrmann, C. Hecht, E. Herdtweck und H.-J. Kneuper, Angew. Chem., 99 (1987) 158; Angew. Chem. Int. Ed. Engl., 26 (1987) 132.

20 B. Schmidkonz, Dissertation, Universität Bayreuth, 1987.

21 L.Y. Goh und T.C.W. Mak, J. Chem. Soc., Chem. Commun., (1986) 1474.

22 Siehe z. B. H. Brunner, H. Kauermann, J. Pfauntsch, J. Wachter, J.H. Enemark und C.G. Young, J. Organomet. Chem., 331 (1987) 45.

23 G. Erker, T. Mühlenbernd, R. Nolte, J.L. Peterson, G. Tainturier und B. Gautheron, J. Organomet. Chem., 314 (1986) C21.

24 P. Cherin und P. Unger, Inorg. Chem., 6 (1967) 1589; O. Foss und V. Janickis, J. Chem. Soc., Dalton Trans., (1980) 624.

25 A. Weiss und H. Witte, Kristallstruktur und chemische Bindung, Verlag Chemie, Weinheim, 1983.

26 M.J. Collins, R.J. Gillespie, J.F. Sawyer und G.J. Schrobilgen, Inorg. Chem., 25 (1986) 2053; D.J. Jones, T. Makani und J. Rozière, J. Chem. Soc., Chem. Commun., (1986) 1275.

27 J. Wachter, J. Coord. Chem. B, 15 (1987) 219.

28 C.-N. Chau, R.W.M. Wardle und J.A. Ibers, Inorg. Chem., 26 (1987) 2740.

29 M. Draganjac und T.B. Rauchfuss, Angew. Chem., 97 (1985) 745; Angew. Chem. Int. Ed. Engl., 24 (1985) 742.

$30 \mathrm{Im}$ Vergleich hierzu lässt sich $\left[\operatorname{Ir}(\mathrm{dpme})_{2} \mathrm{Se}_{4}\right]^{+}$mit $\mathbf{P P h}_{3}$ bereits bei Raumtemperatur zu [Ir(dpme) $\left.{ }_{2} \mathrm{Se}_{2}\right]^{+}$abbauen: A.P. Ginsberg, J.H. Osborne und C.R. Sprinkle, Inorg. Chem., 22 (1983) 1781.

31 C. Burschka, K. Leonhard und H. Werner, Z. Anorg. Allg. Chem., 464 (1980) 30.

32 Y. Wakatsuki und H. Yamazaki, J. Organomet. Chem., 64 (1974) 393. 
33 A. Meerschaut, P. Grenouilleau, P. Brec, M. Evain und J. Rouxel, J. Less-Common Met., 116 (1986) 229.

34 F. Fehér, in G. Brauer (Hrsg.), Handbuch der Präparativen Anorganischen Chemie, Enke Verlag, Stuttgart, 1981, S. 411.

35 W.J. Bailey Jr., D.M. Collins, F.A. Cotton, J.C. Baldwin und W.C. Kaska, J. Organomet. Chem., 165 (1979) 373; R. Ginsburg, L.M. Cirjak und L.F. Dahl, J. Chem. Soc., Chem. Commun., (1979) 468.

36 Weitere Einzelheiten zur Kristallstrukturuntersuchung können bei den Autoren angefordert werden. 